\title{
MicroRNA-26b inhibits the tumor growth of human liver cancer through the PI3K/Akt and NF-кB/MMP-9/VEGF pathways
}

\author{
YAN FENG ${ }^{1}$, LI-LI ZU ${ }^{2}$ and LEI ZHANG ${ }^{3}$ \\ ${ }^{1}$ Department of Anesthesiology, The First Affiliated Hospital of Dalian Medical University; \\ ${ }^{2}$ Department of Ultrasound, The Affiliated Zhongshan Hospital of Dalian University; \\ ${ }^{3}$ Department of Emergency, The First Affiliated Hospital of Dalian Medical University, \\ Dalian, Liaoning 116011, P.R. China
}

Received December 13, 2016; Accepted February 22, 2018

DOI: $10.3892 /$ or.2018.6289

\begin{abstract}
We investigated the role of microRNA-26b signaling in mediating the tumor growth of human liver cancer. MicroRNA-26b expression was observably downregulated in human liver cancer tissue. Forced upregulation of microRNA26b had anticancer effects, inhibited cell proliferation, induced apoptosis and promoted Bax and caspase-3/-9 protein expression in a liver cancer cell line as determined by MTT assay, flow cytometry and western blot analysis. MicroRNA-26b significantly suppressed the PI3K/Akt and NF-кB/MMP-9/ VEGF pathways. PI3K inhibitor significantly facilitated the effects of microRNA-26b regarding the suppression of the PI3K/Akt pathway, inhibition of cell proliferation, induction of apoptosis and increases in Bax and caspase-3/-9 protein expression in the liver cancer cell line. NF- $\mathrm{kB}$ inhibitor also significantly enhanced the effects of microRNA-26b regarding suppression of the NF- $\kappa \mathrm{B} / \mathrm{MMP}-9 / \mathrm{VEGF}$ pathway, inhibition of cell proliferation, induction of apoptosis, and promotion of Bax and caspase-3/-9 protein expression in the liver cancer cell line. The present study demonstrated that microRNA-26b inhibits the tumor growth of human liver cancer through the PI3K/Akt and NF-kB/MMP-9/VEGF pathways.
\end{abstract}

\section{Introduction}

Liver cancer is the major type of primary liver cancer as well as one of the most common malignant tumors worldwide (1). China is a country with the highest incidence of liver cancer in the world and its overall morbidity and mortality

Correspondence to: Dr Lei Zhang, Department of Emergency, The First Affiliated Hospital of Dalian Medical University, 222 Zhongshan Road, Dalian, Liaoning 116011, P.R. China

E-mail: leizhang1967@163.com

Key words: microRNA-26b, liver cancer, PI3K/Akt, NF-кB/ MMP-9/VEGF show increasing trends (2). Although therapeutic approaches targeted at liver cancer have been continuously advanced in recent years, the overall therapeutic effects on liver cancer have shown no obvious improvement, and recurrence and metastasis remain the major factors affecting the prognosis of patients with liver cancer (3).

Tumor metastasis is a multi-step, multi-stage and multipathway complex process, which includes the detachment of tumor cells from the primary lesion, invasion into blood vessels or lymph vessels, migration and adhesion to appropriate sites, induction of tumor angiogenesis, anti-host antitumor immunity, and the eventual formation of distal metastasis (4). Multiple genes are involved during this process. A vast majority of current studies have focused on the effects and functions of related genes in tumor invasion and metastasis, such as oncogenes, tumor-suppressor genes and pro-metastatic genes (5). Research has demonstrated that, apart from these protein-coding genes which can regulate tumor invasion and metastasis, various non-coding genes, particularly microRNA molecules, are also involved in regulating tumor invasion and metastasis. MicroRNAs have been found to be abnormally expressed in multiple malignant tumors, including colorectal, lung, breast, liver, brain and prostate cancer, and they play an important role in tumor proliferation, differentiation, invasion, metastasis and treatment response (6).

The PI3K/Akt signaling pathway plays a vital role in the genesis, development and survival of tumors (7). With multiple biological activities, activated Akt can catalyze the phosphorylation of a series of proteins, promote tumor cell growth and proliferation, inhibit apoptosis, facilitate invasion and metastasis, regulate endothelial growth and angiogenesis, and increase sensitivity to radiation. PTEN molecule is a significant inhibitory regulator of PI3K (8).

As is indicated in research, PTEN gene deletions or mutations can be observed in multiple human malignant solid tumors (including prostate cancer, glioblastoma multiforme, melanoma, thyroid cancer and bladder cancer) as well as hereditary tumor susceptible syndrome (7). The PTEN gene exerts certain effects on regulating phosphatase activities of multiple intracellular protein molecules, rendering gene deletion and mutation, which plays an important role in tumor genesis and development (9). 
As a type of nuclear transcription factor that is extensively distributed in multiple cells, $\mathrm{NF}-\kappa \mathrm{B}$ plays an important role in aspects of cell carcinogenesis and apoptosis regulation (10). It has been indicated in research that the $N F-\kappa B$ signal transduction pathway is involved in the transcription expression of oncogenes and tumor-suppressor genes in the liver, which can participate in the genesis and development of liver cancer by inhibiting cell apoptosis. As a type of polyphonic transcription factor with multi-function, $\mathrm{NF}-\kappa \mathrm{B}$ can regulate cell apoptosis- and proliferation-associated genes, thus playing an important role in cell carcinogenesis (11). The NF- $\mathrm{NB}$ protein family is comprised of 5 distinct subunits, among which, the p50/p65 heterodimer is the most common one. Abnormal regulation is induced when $\mathrm{NF}-\kappa \mathrm{B}$ is inappropriately activated and located at the cell nucleus where it cannot return to the cytoplasm (12). Furthermore, we examined the role of microRNA-26b signaling in mediating the tumor growth of human liver cancer.

\section{Materials and methods}

Liver cancer tissue. Written informed consent was obtained from all patients according to the guidelines approved by the Institutional Research Board of The First Affiliated Hospital of Dalian Medical University and information regarding the clinical samples are shown at Table I. Liver cancer tissue and the adjacent normal tissue were collected from patients at The First Affiliated Hospital of Dalian Medical University from May 2016 to August 2016. All specimens were immediately frozen in liquid nitrogen and stored at $-70^{\circ} \mathrm{C}$ until use.

$q R T-P C R$ detection. Total RNA isolation from tissues was conducted using the TRIzol kit (Invitrogen, Carlsbad, CA, USA), followed by RNeasy Mini kit (Qiagen, Inc., Valencia, CA, USA). RNA (100 ng) was reverse-transcribed into cDNA using an iScript cDNA synthesis kit (Bio-Rad Laboratories, Inc., Hercules, CA, USA). MicroRNA-26b expression was quantified using miR-qRT PCR using the Hairpin- $\mathrm{it}^{\mathrm{TM}}$ miRNA qPCR Quantitation kit (GenePharma Co., Ltd., Shanghai, China) and measured as using the $2^{-\Delta \Delta \mathrm{Ct}}$ method.

HepG2 cell culture and controlled microRNA-26 expression. HepG 2 cells were obtained from the Cell Culture Center of Chinese Peking Union Medical College (Beijing, China) and cultured in Dulbecco's modified Eagle's medium (DMEM) containing 10\% fetal bovine serum (FBS) (All from Thermo Fisher Scientific, Inc., Shanghai, China) at $37^{\circ} \mathrm{C}$ in $5 \% \mathrm{CO}_{2}$. Anti-microRNA-26 and control inhibitor mimics were purchased from RiboBio Co., Ltd. (Guangzhou, China). MicroRNA-26 and control mimics were transfected into HepG2 cells using Invitrogen ${ }^{\mathrm{TM}}$ Lipofectamine 2000 reagent (Thermo Fisher Scientific). After cells were transfected for $6 \mathrm{~h}$, the cells was treated with $10 \mu \mathrm{M}$ of 3-methyladenine (PI3K inhibitor) or $5 \mu \mathrm{M}$ of PDTC (NF- $\kappa \mathrm{B}$ inhibitor) for $48 \mathrm{~h}$.

Proliferation assay. Cells transfected with the mimics $\left(1 \times 10^{5}\right)$ $\mathrm{ml})$ were added to each well of a 96-well plate for 24,48 and $72 \mathrm{~h}$. MTT $(20 \mu \mathrm{l})(5 \mathrm{mg} / \mathrm{ml}$; Sigma-Aldrich China, Inc., Shanghai, China) was added and incubation was carried our
Table I. Information concerning the clinical samples from the patients with liver cancer.

\begin{tabular}{lcc}
\hline Variables & All patients $(\mathrm{N}=54)$ & P-value \\
\hline Age (years) & 32 & 0.592 \\
$\leq 60$ & 22 & \\
$>60$ & & 0.321 \\
Sex & 30 & \\
Female & 24 & \\
Male & & 0.832 \\
Tumor size (cm) & 25 & \\
$\leq 3.0$ & 29 & \\
$>3.0$ & & \\
Edmondson grade & 11 & \\
I-II & 43 & \\
III-IV & & \\
\hline
\end{tabular}

at $37^{\circ} \mathrm{C}$ for $4 \mathrm{~h}$ and $150 \mu \mathrm{l}$ DMSO was added and agitated for $20 \mathrm{~min}$ in the dark for dissolution. The optical density (OD) was then measured at an absorbance of $570 \mathrm{~nm}$.

Apoptosis assay. Cells transfected with the mimics $\left(1 \times 10^{5} / \mathrm{ml}\right)$ were added to each well of a 6-well plate for $48 \mathrm{~h}$. Cells were washed twice with PBS, and then centrifuged at 1,000 $\mathrm{x} g$ for $5 \mathrm{~min}$. Annexin V/FITC $(10 \mu \mathrm{l})$ and propidium iodide (PI) $(5 \mu \mathrm{l})$ (Becton-Dickinson, San Jose, CA USA) were added and maintained at room temperature for $15 \mathrm{~min}$ in the dark. Apoptosis were then analyzed by flow cytometry (BD FACSCalibur; BD Biosciences, Franklin Lakes, NJ, USA).

Western blotting. Cells transfected with the mimics $\left(1 \times 10^{5} / \mathrm{ml}\right)$ were added to each well of a 6-well plate for $48 \mathrm{~h}$, and then incubated with RIPA lysis buffer (Beyotime, Inc., Jiangsu, China) for $30 \mathrm{~min}$. Protein contents were quantified using the Bicinchoninic acid (BCA) protein kit (Beyotime, Inc.). Protein $(50 \mu \mathrm{g})$ was separated by $8-12 \%$ sodium dodecyl sulfate polyacrylamide gel electrophoresis (SDS-PAGE) and transferred into a polyvinylidene fluoride (PVDF) membrane. The membrane was incubated with anti-PI3K (4249), anti-p-Akt (4060), anti-NF-кB (8242), anti-MMP-9 (13667), anti-pVEGF (2463) and anti-GAPDH (5174) (Cell Signaling Technology, Inc., Danvers, MA, USA) antibodies overnight at $4^{\circ} \mathrm{C}$. The membrane was then incubated with the anti-rabbit secondary antibody at $37^{\circ} \mathrm{C}$ for $1 \mathrm{~h}$ and protein bands were visualized using an enhanced chemiluminesecence system.

Statistical analysis. Data are shown as mean \pm SEM. The correlations were analyzed using the analysis of variance (ANOVA). A probability value of $<0.05$ was chosen for statistical significance.

\section{Results}

MicroRNA-26b expression in human liver cancer tissue. To analyze the clinical samples of human liver cancer tissue 


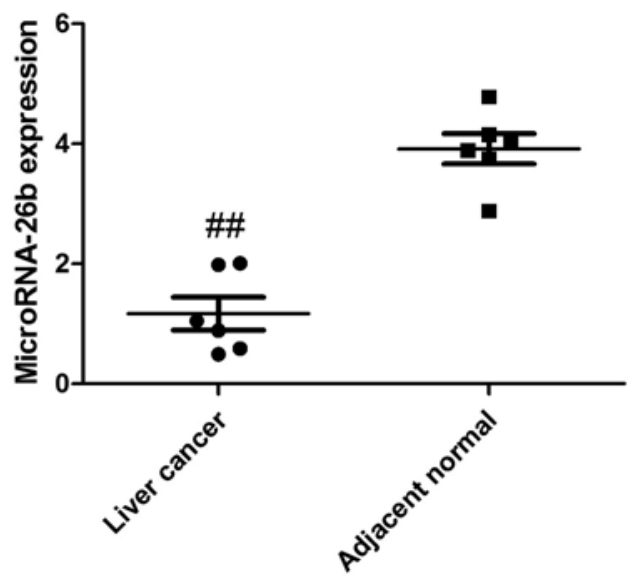

Figure 1. MicroRNA-26b expression in human liver cancer tissues; ${ }^{\# \#} \mathrm{P}<0.01$ vs. the control group.

and the adjacent normal tissue, we performed qRT-PCR to detect the expression of microRNA-26b. As shown in Fig. 1, microRNA-26b expression was observably downregulated in yhe human liver cancer tissues, compared with that noted in the adjacent normal tissues.

Correlation between microRNA-26b and cell growth of liver cancer. In order to assess whether microRNA-26b possess anticancer effects on liver cancer, microRNA-26b expression was upregulated in HepG2 cells using microRNA-26b mimics. Fig. 2 indicates that microRNA-26b was upregulated in the HepG2 cells using microRNA-26b mimics, and upregulation of microRNA-26b significantly decreased cell proliferation and induced apoptosis of the HepG2 cells.

Correlation between microRNA-26b and Bax and caspase-3/-9 protein expression in liver cancer. Then, we analyzed the correlation between microRNA-26b and Bax and caspase-3/-9 protein expression in liver cancer. As shown in Fig. 3, statistical analyses demonstrated that microRNA-26b significantly induced Bax and caspase-3/-9 protein expression in the HepG2 cells.

Correlation between microRNA-26b and PI3K/Akt and $N F-\kappa B / M M P-9 / V E G F$ pathways. To investigate the correlation between microRNA-26b and PI3K/Akt and NF- $\kappa \mathrm{B} /$ MMP-9/VEGF pathways. PI3K, p-Akt, NF- $\mathrm{B}, \mathrm{MMP}-9$ and VEGF protein levels were significantly suppressed in the HepG2 cells by the upregulation of microRNA-26b, compared with the negative control group (Figs. 4 and 5). These results suggest that microRNA-26b may have a functional role in the development of human liver cancer.

PI3K inhibitor regulates the anticancer effects of microRNA$26 b$ on PI3K/Akt pathways of liver cancer. We examined whether the function of PI3K participates in the anticancer effects of microRNA-26b on liver cancer. PI3K inhibitor significantly further suppressed PI3K and p-Akt protein expression in the HepG2 cells by microRNA-26b, when compared with the negative control group (Fig. 6).

PI3K inhibitor regulates the anticancer effects of microRNA$26 b$ on liver cancer cell growth. We further examined the
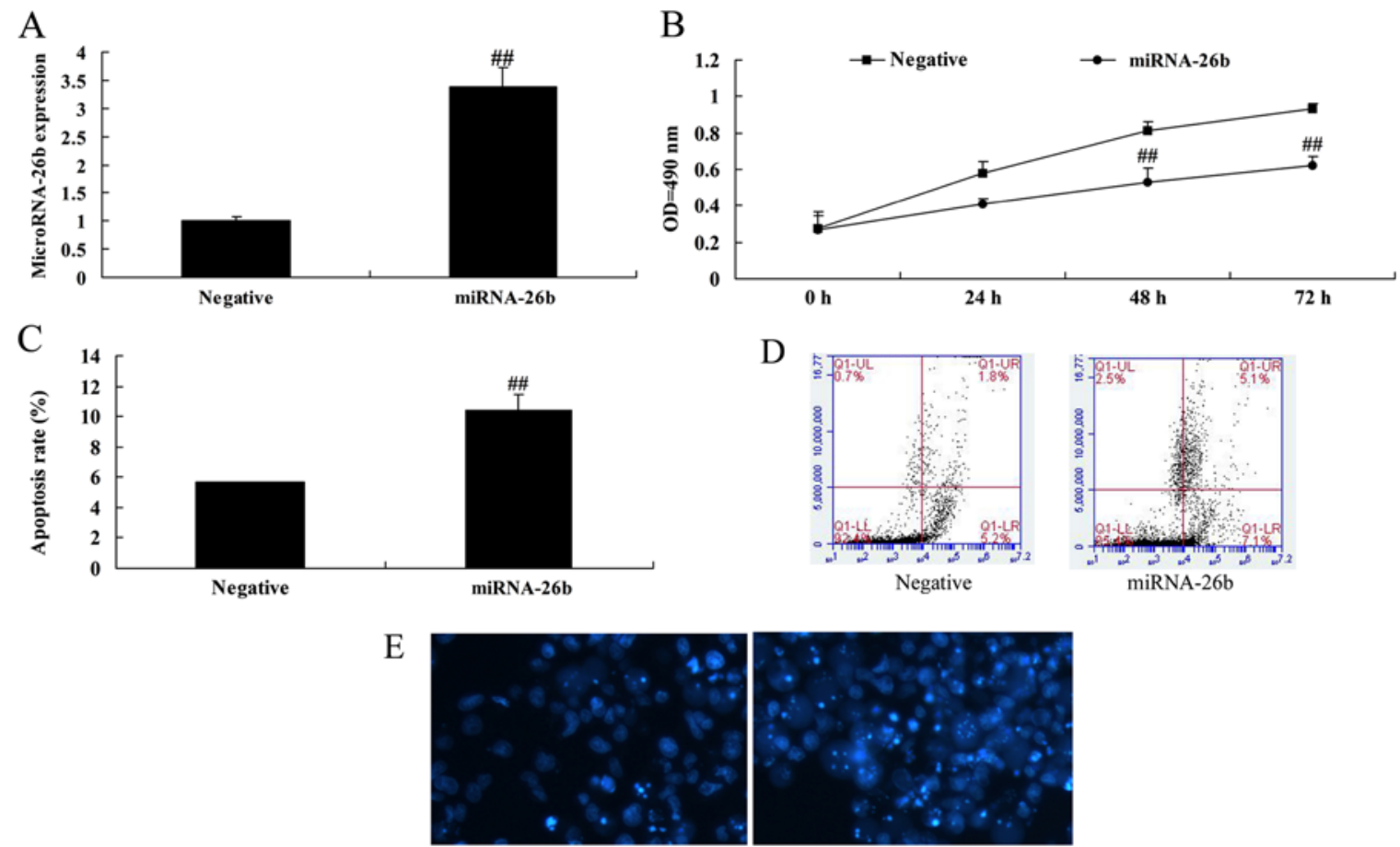

Negative

miRNA-26b

Figure 2. Effect of the microRNA-26b mimic on the growth of HepG2 liver cancer cells. (A) microRNA-26b expression in HepG2 cells transfected with the microRNA-26b mimics. Effects of microRNA-26b mimic on (B) cell growth by MTT assay and (C and D) apoptosis by flow cytometry and (E) DAPI staining assay; ${ }^{\# \#} \mathrm{P}<0.01$ vs. negative control group. 
A

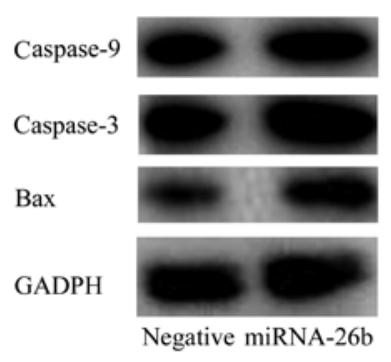

$\mathrm{C}$

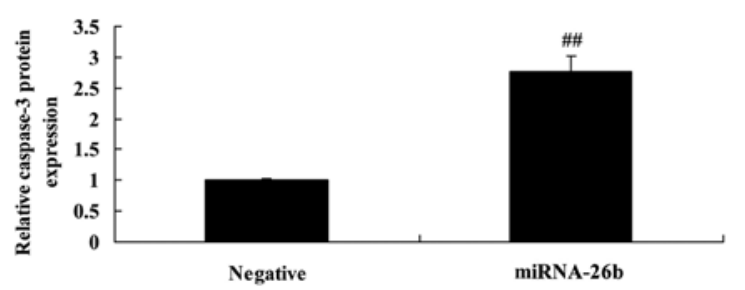

B

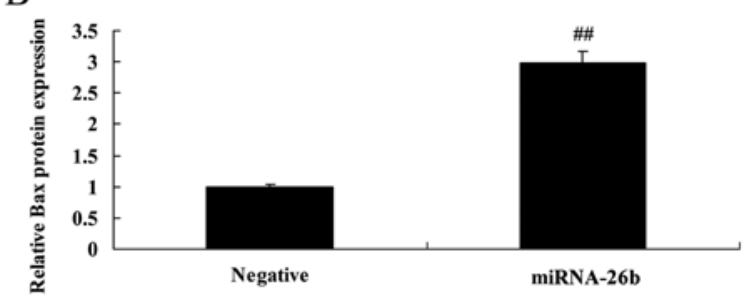

$\mathrm{D}$

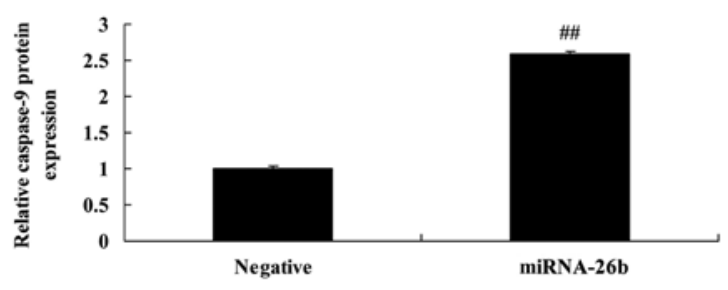

Figure 3. Effect of microRNA-26b mimic on Bax and caspase-3/-9 protein expression in liver cancer HepG2 cells. Effect of microRNA-26b on Bax and caspase-3/-9 protein expression by (A) western blot analysis and (B-D) statistical analyses of Bax and caspase-3/-9 protein expression in liver cancer cells; ${ }^{\# \#} \mathrm{P}<0.01$ vs. negative control group.
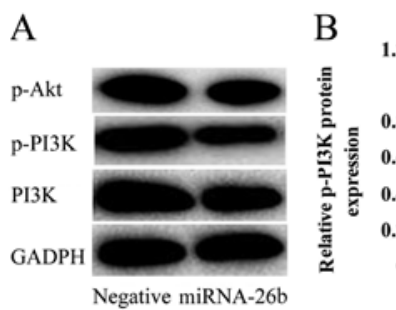

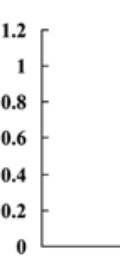

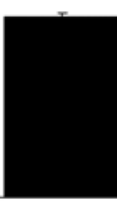

Negative

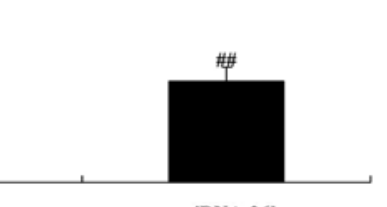

miRNA-26b

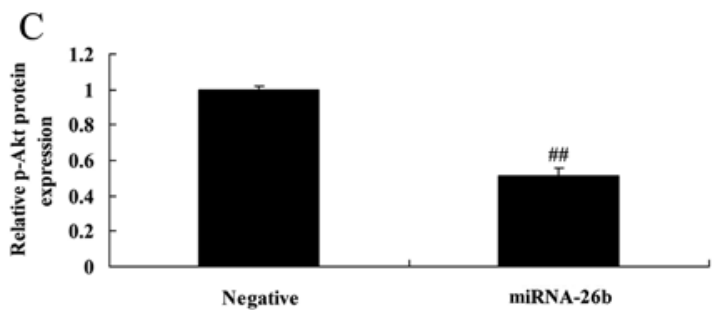

Figure 4. Effect of microRNA-26b mimic on the PI3K/Akt pathways in HepG2 cells. Effect of microRNA-26b on PI3K and p-Akt protein expression by (A) western blot analysis and (B and C) statistical analyses of PI3K and p-Akt protein expression in liver cancer cells; ${ }^{\# \#} \mathrm{P}<0.01$ vs. negative control group.

A

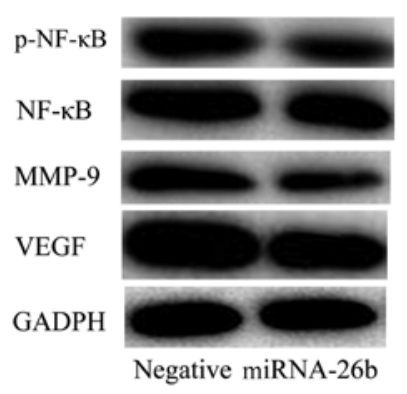

$\mathrm{C}$

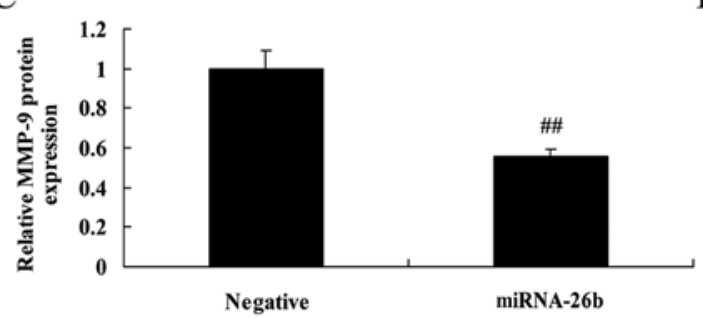

B

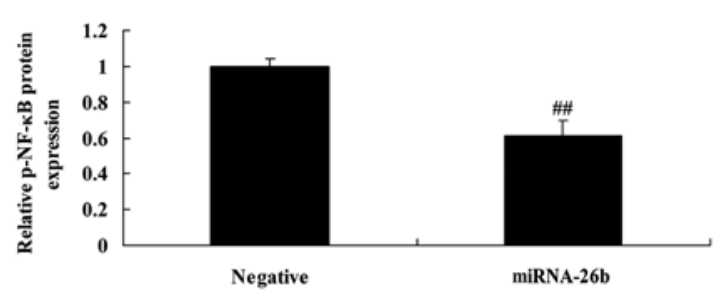

D

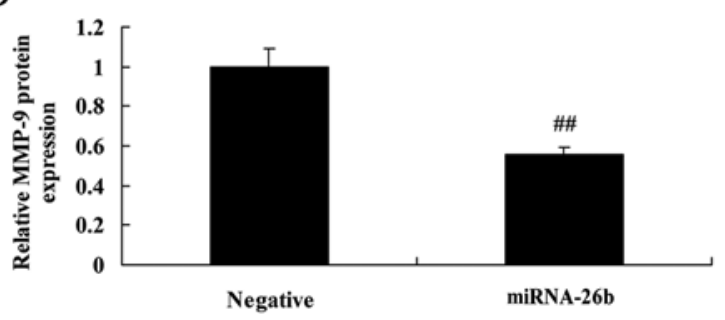

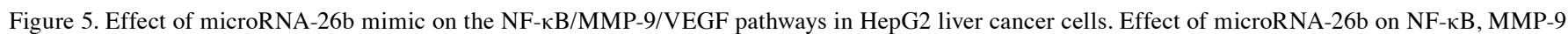

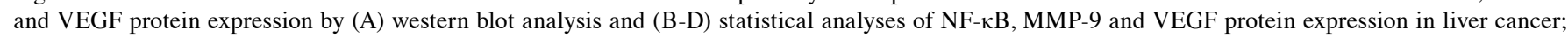
${ }^{\# \#} \mathrm{P}<0.01$ vs. negative control group.

function of PI3K in the anticancer effects of microRNA-26b on liver cancer cell growth and apoptosis. Analysis of MTT assay and flow cytometry showed that, as compared to the negative control, the PI3K inhibitor significantly accelerated 
A

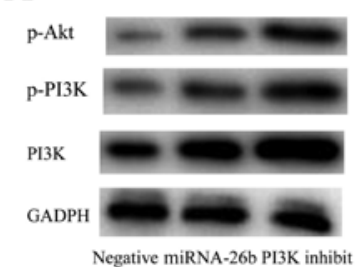

B

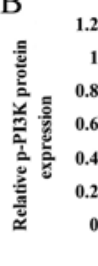

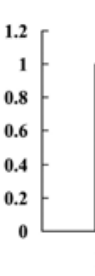

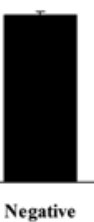

Negative
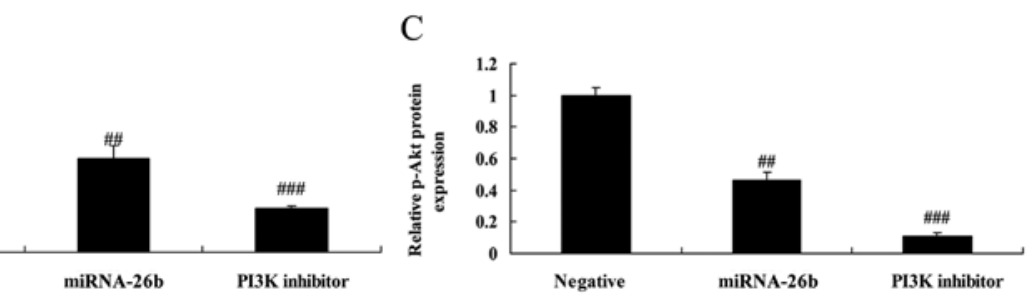

Figure 6. PI3K inhibitor regulates the anticancer effects of microRNA-26b on PI3K/Akt pathways of liver cancer. PI3K inhibitor regulates the anticancer effects of microRNA-26b on PI3K and p-Akt protein expressions by (A) western blot and (B and C) statistical analyses PI3K and p-Akt protein expressions of liver cancer; ${ }^{\# \#} \mathrm{P}<0.01$ vs. control group, ${ }^{\# \#} \mathrm{P}<0.01$ vs. microRNA-26b group.

A

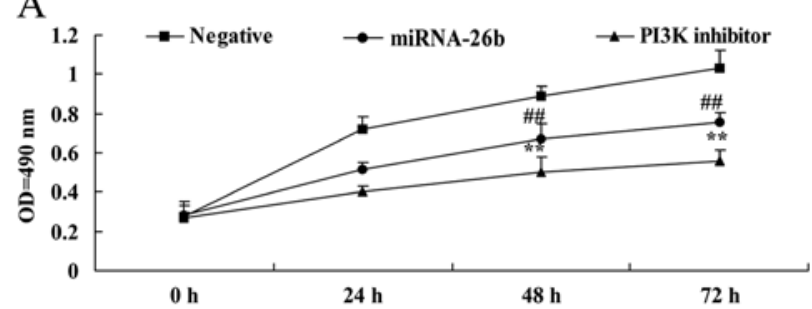

C

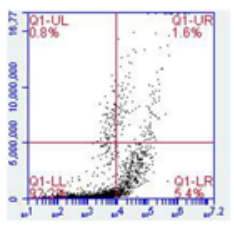

Negative

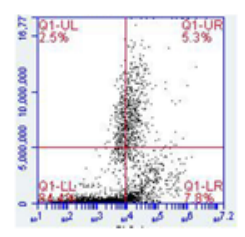

miRNA-26b

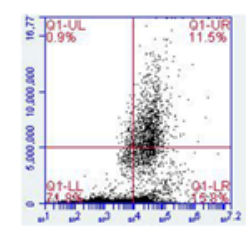

PI3K inhibitor

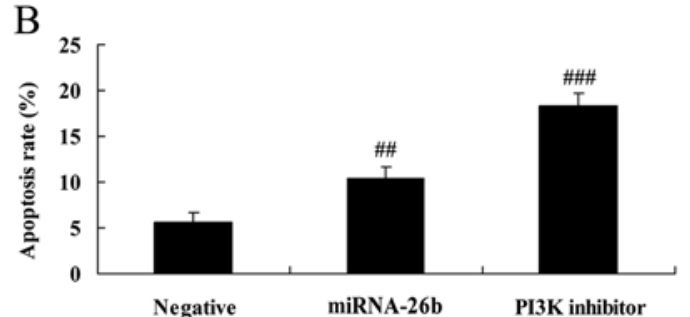

D

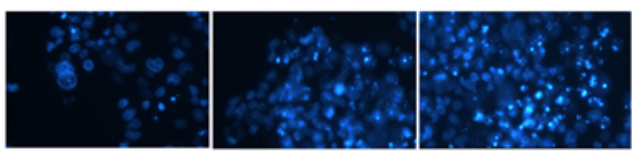

Negative
miRNA-26b

Figure 7. PI3K inhibitor regulates the anticancer effects of microRNA-26b in liver cancer cell growth. PI3K inhibitor regulates the anticancer effects of microRNA-26b on (A) liver cancer cell growth as determined by MTT assay and (B and C) apoptosis by flow cytometry and (D) DAPI staining assay; ${ }^{\#} \mathrm{P}<0.01$ vs. negative control group, ${ }^{\# \#} \mathrm{P}<0.01$ vs. microRNA-26b group.

A

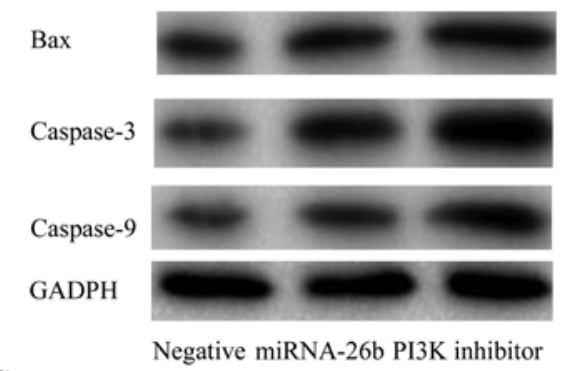

$\mathrm{C}$

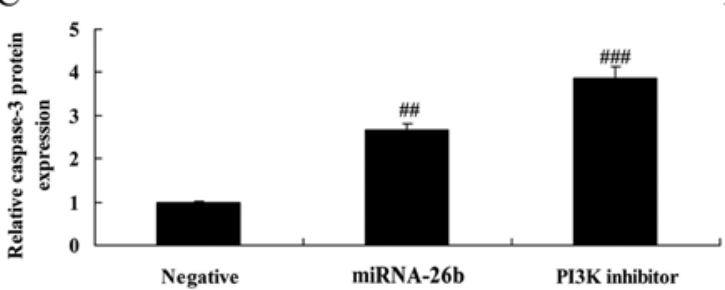

B

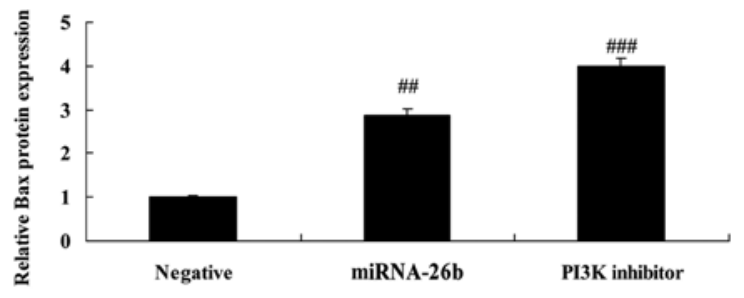

D

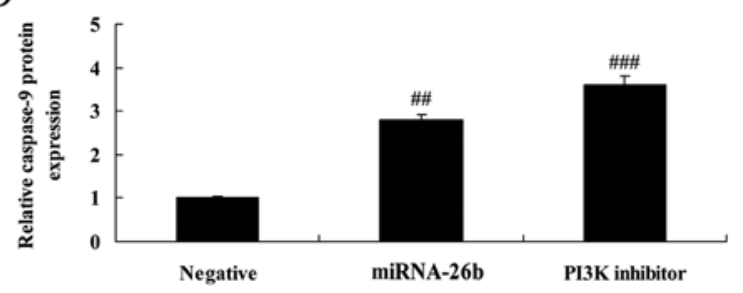

Figure 8. PI3K inhibitor regulates the anticancer effects of microRNA-26b on Bax and caspase-3/-9 protein expression in liver cancer HepG2 cells. PI3K inhibitor regulates the anticancer effects of microRNA-26b on Bax and caspase-3/-9 protein expression by (A) western blot analysis and (B-D) statistical analyses of Bax and caspase-3/-9 protein expression in liver cancer; ${ }^{\# \#} \mathrm{P}<0.01$ vs. negative control group, ${ }^{\# \#} \mathrm{P}<0.01$ vs. microRNA-26b group.

the anticancer effects of microRNA-26b on the inhibition of liver cancer cell growth and induction of apoptosis (Fig. 7).
PI3K inhibitor regulates the anticancer effects of microRNA$26 b$ on Bax and caspase-3/-9 protein expression in liver 
A

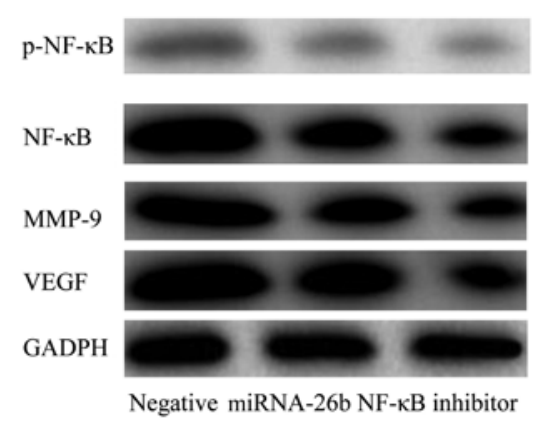

$\mathrm{C}$

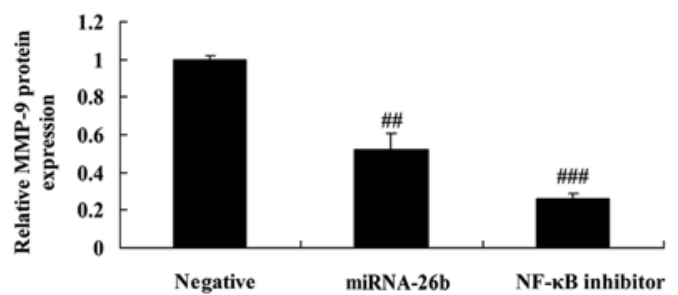

B

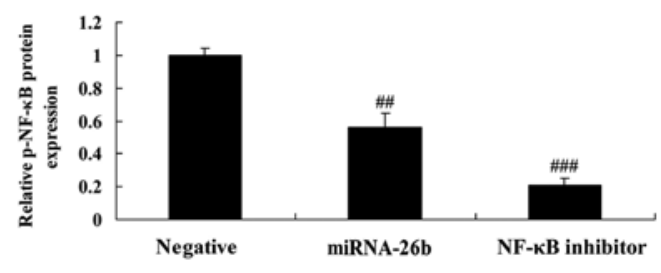

D

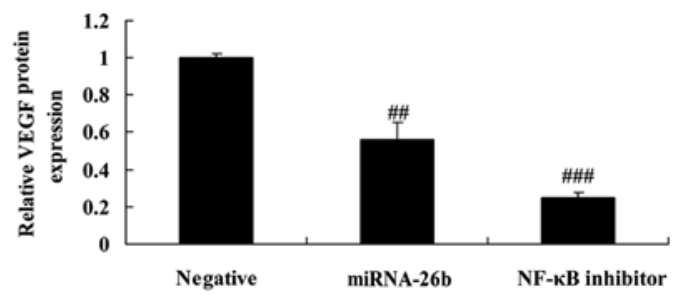

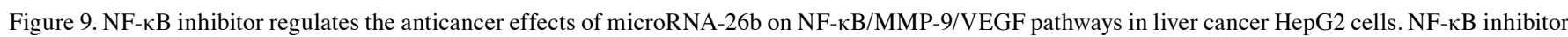
regulates the anticancer effects of microRNA-26b on NF- $\mathrm{BB}$, MMP-9 and VEGF protein expression by (A) western blot analysis and (B-D) statistical analyses of NF- $\mathrm{KB}, \mathrm{MMP}-9$ and VEGF protein expression in liver cancer. ${ }^{\# \#} \mathrm{P}<0.01$ vs. negative control group, ${ }^{\# \# "} \mathrm{P}<0.01$ vs. microRNA-26b group.

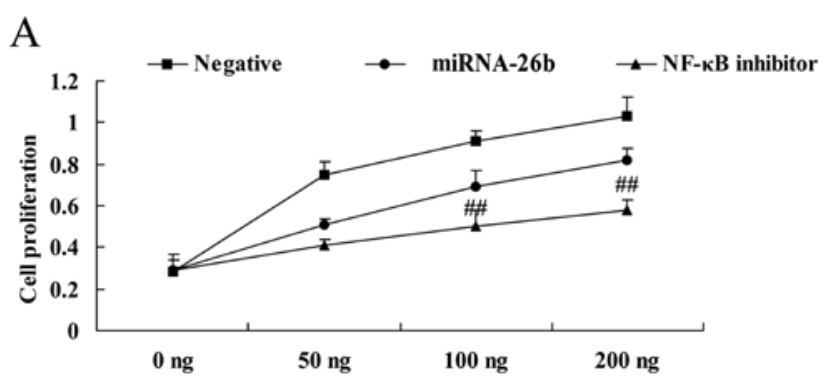

C

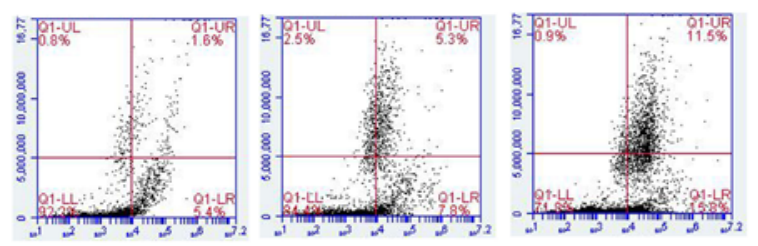

B

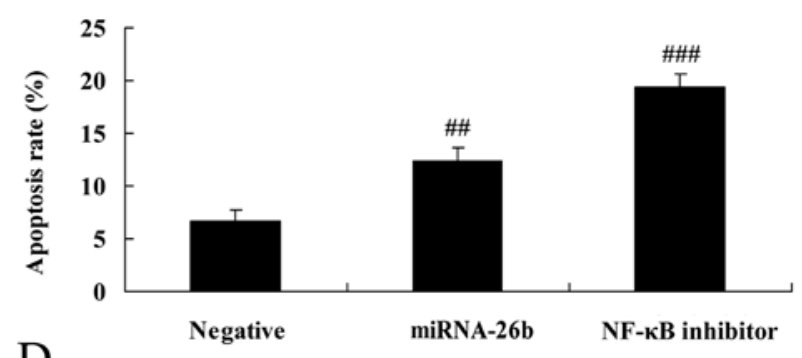

D

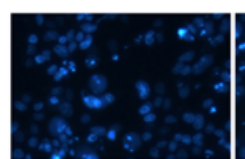

Negative

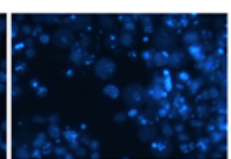

miRNA-26b

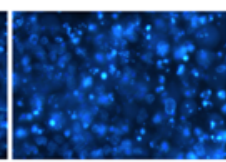

NF-кB inhibitor

Figure 10. NF- $\kappa$ B inhibitor regulates the anticancer effects of microRNA-26b on liver cancer cell growth. NF- $\kappa$ B inhibitor regulates the anticancer effects of microRNA-26b on (A) liver cancer cell growth by MTT assay and (B and C) apoptosis by flow cytometry and (D) DAPI staining assay; ${ }^{\#}$ P $<0.01$ vs. control group, ${ }^{\# \#} \mathrm{P}<0.01$ vs. microRNA-26b group.

cancer. We analyzed the effects of PI3K on Bax and caspase3/-9 protein levels in the HepG2 cells by microRNA-26b using western blotting. The PI3K inhibitor further significantly induced Bax and caspase-3/-9 protein expression in the liver cancer cells by microRNA-26b, compared with the negative control group (Fig. 8). These data demonstrated that PI3K is correlated with the anticancer effects of microRNA-26b on liver cancer.

$N F-\kappa B$ inhibitor regulates the anticancer effects of microRNA$26 b$ on $N F-\kappa B / M M P-9 / V E G F$ pathways in liver cancer. We examined whether NF- $\kappa \mathrm{B}$ influences the anticancer effects of microRNA-26b in liver cancer. The NF- $\kappa B$ inhibitor significantly further suppressed NF- $\kappa$ B, MMP-9 and VEGF protein expression in liver cancer cells by microRNA-26b, compared with the negative control group (Fig. 9).

$N F-\kappa B$ inhibitor regulates the anticancer effects of microRNA-26b on liver cancer cell growth. The results of analyses for liver cancer cell growth and apoptosis using MTT assay and flow cytometry in HepG2 cells by microRNA-26b and $N F-\kappa B$ inhibitor are shown in Fig. 10. It was observed that $\mathrm{NF}-\kappa \mathrm{B}$ inhibitor significantly facilitated the anticancer effects of microRNA-26b on liver cancer cell growth inhibitor and apoptosis induction (Fig. 10).

$N F-\kappa B$ inhibitor regulates the anticancer effects of microRNA$26 b$ on Bax and caspase-3/-9 protein expression in liver 
A

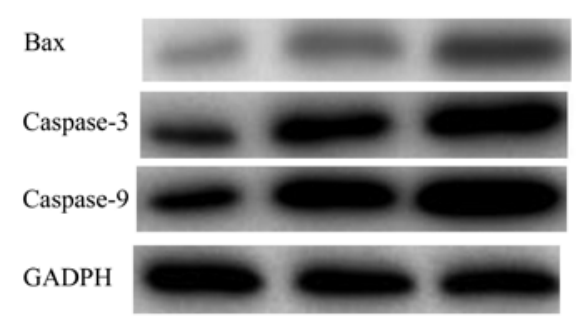

Negative miRNA-26b NF- $\kappa$ B inhibitor

$\mathrm{C}$

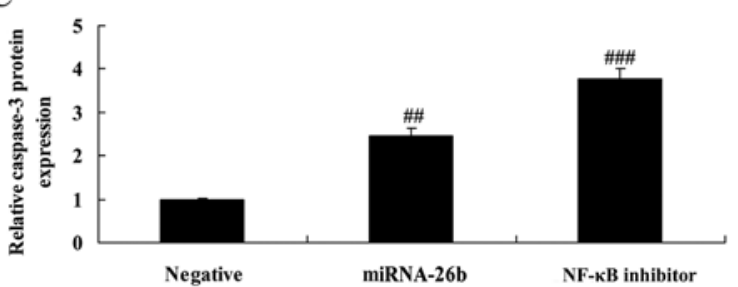

B

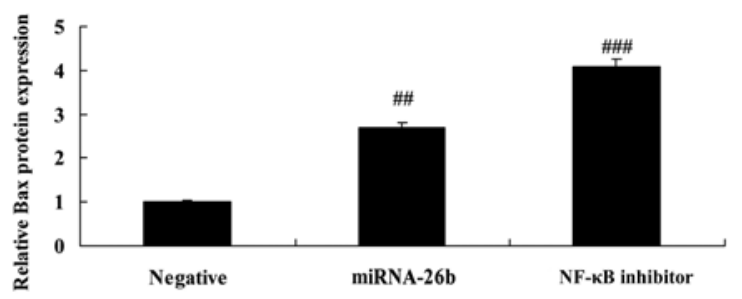

D

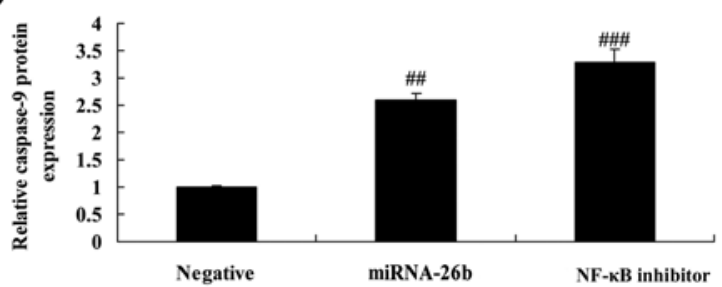

Figure 11. NF- $\mathrm{kB}$ inhibitor regulates the anticancer effects of microRNA-26b on Bax and caspase-3/-9 protein expression in liver cancer HepG2 cells. NF- $\mathrm{kB}$ inhibitor regulates the anticancer effects of microRNA-26b on Bax and caspase-3/-9 protein expression by (A) western blot analysis and (B-D) statistical analyses of Bax and caspase-3/-9 protein expression in liver cancer. ${ }^{\# \#} \mathrm{P}<0.01$ vs. negative control group, ${ }^{\# \#} \mathrm{P}<0.01$ vs. microRNA-26b group.

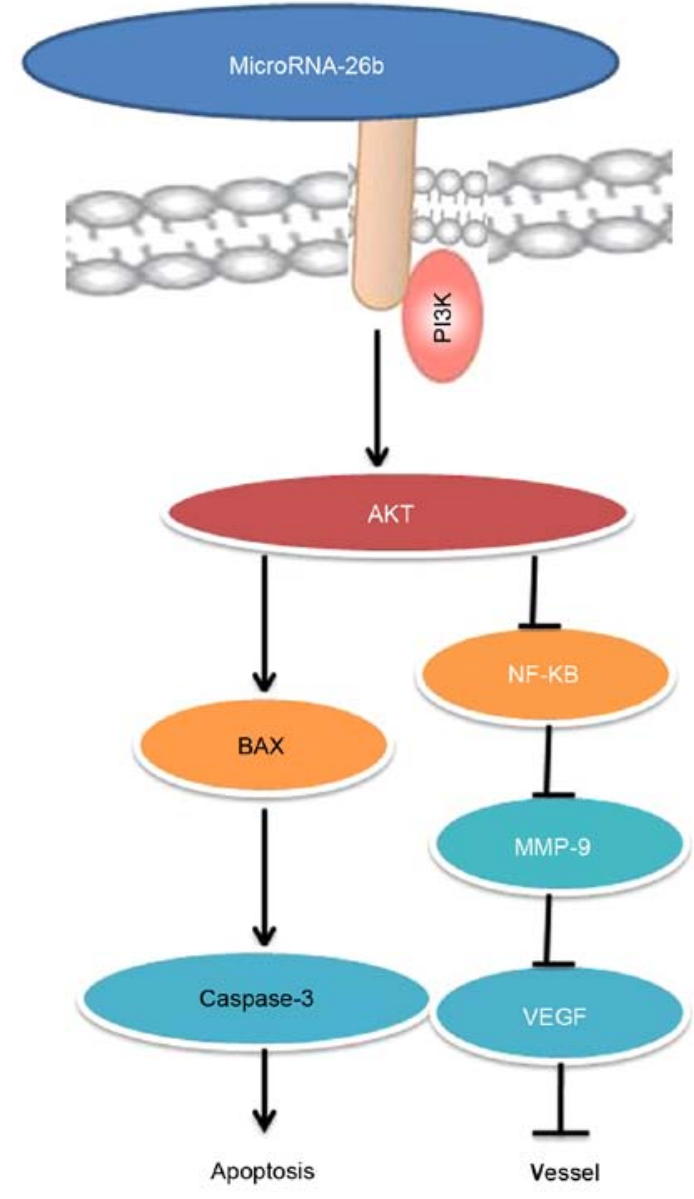

Figure 12. MicroRNA-26b inhibits tumor growth of human liver cancer through the PI3K/Akt and NF-kB/MMP-9/VEGF pathways.

cancer. In addition, we analyzed Bax and caspase-3/-9 protein expression in liver cancer by microRNA-26b. As shown in Fig. 11, NF- $\mathrm{BB}$ inhibitor significantly further induced Bax and caspase-3/-9 protein expression in the HepG2 cells by microRNA-26b, compared with the negative control group.

\section{Discussion}

Liver cancer is a major disease that severely threatens human health. Liver cancer shows an annually increasing trend due to the influence of factors such as viral hepatitis and environment (13). Although liver cancer is treated by conventional surgery combined with drugs (chemotherapy) in the clinic, recurrence and metastasis, together with the resistance of cancer cells to drugs has resulted in bleak prospects for treating liver cancer. Consequently, unveiling the molecular mechanisms of the drug resistance of liver cancer will bring new hopes for treating liver cancer (14). In the present study, we showed that microRNA-26b expression was observably downregulation in human liver cancer tissue.

microRNAs exert effects that are analogous to tumor suppressors or oncogenes by binding with oncogenes or tumor suppressor genes, and are closely related to tumor genesis and development (15). Participating in tumor invasion and metastasis, microRNAs play a regulatory role in multiple steps during tumor metastasis (16). Abnormal microRNA expression has been reported e to exist in human cancers, and the differential expression profile has been reported in multiple cancers successively, including breast, colorectal and liver cancer (17). microRNAs are located in tumor-associated fragile sites in the genome, prone to develop deletion, amplification or translocation. microRNAs exert effects that are analogous to tumor suppressors or oncogenes through locating in oncogene or tumor suppressor gene (18). These data demonstrated that microRNA-26b has anticancer effects, by inhibiting cell proliferation and inducing apoptosis of liver cancer cells.

$\mathrm{Bcl}-2$ protein can form a heterodimer with the Bax protein (the pro-apoptotic factor), which inhibits tumor apoptosis, prolongs the cell lifespan, and thus participates in tumor 
genesis and development (14). Overexpression of Bcl-2 is significantly correlated with radiotherapy resistance of laryngeal squamous cell carcinoma, and it can also prompt the proliferation of tumor cells whose DNA has been damaged by radiation (8). In the present study, microRNA-26b promoted Bax and caspase-3/-9 protein expressions in liver cancer cells. MicroRNA-26b had anticancer effects by inhibiting cell proliferation, inducing apoptosis and promoting Bax and caspase-3/-9 protein expressions in a liver cancer cell line. MicroRNA-26b significantly suppressed PI3K/Akt and NF- $\kappa$ B/MMP-9/VEGF pathways.

The PI3K/Akt signaling pathway plays a vital role during tumor genesis and development. Possessing multiple biological activities, the activated Akt catalyzes the phosphorylation of a series of proteins, promotes tumor cell growth and proliferation, inhibits apoptosis, enhances invasion and metastasis, regulates endothelial growth and angiogenesis, and increases sensitivity to radiation (8). AKT, an important substance for transmitting growth signals, plays a role of central information substance during the growth of tumor cells; therefore, AKT activation predicts exuberant tumor cell growth. PTEN is considered to be an extremely important tumor-suppressor gene, the C2 domain of which plays a crucial role in the process of inhibiting tumor growth and metastasis (19). The results indicated that microRNA-26b significantly suppressed the PI3K/Akt pathway. Suppression of PI3K significantly facilitated the effects of microRNA-26b on suppression of the $\mathrm{PI} 3 \mathrm{~K} / \mathrm{Akt}$ pathway, inhibition of cell proliferation, induction of apoptosis and increases in Bax and caspase-3/-9 protein expression in a liver cancer cell line. Zhu et al demonstrated that microRNA-26b participates in regulating the chemotactic response of mesenchymal stem cells (MSCs) toward hepatocyte growth factor through activation of Akt and FAK (20).

$\mathrm{NF}-\kappa \mathrm{B}$ is a type of heterodimer composed by $50 \mathrm{ku}$ and65 ku protein, which forms a compound with its inhibitor I $\mathrm{Bs}$ under normal conditions, and remains in the resting state (21). When stimulated by external factors, such as hypoxia, cytokine, viral protein, mitogen and ultraviolet, I $\kappa$ Bs may be degraded, and $\mathrm{I} \kappa \mathrm{Bs}$ in the trimer compound will be phosphorylated, thus leading to dissociation with

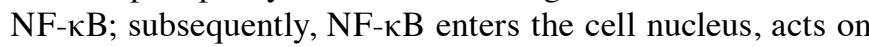
the target factor and exerts its function $(21,22) . N F-\kappa B$ is a type of transcription factor with multidirectional regulatory function, which can regulate the expression of multiple genes, such as apoptosis-associated genes, oncogenes, tumor metastasis-associated adhesion molecules, and extracellular matrix protease; particularly, it can upregulate the expression of VEGF and MMP-9 genes, and is closely associated with tumor genesis, infiltration and metastasis (22). In addition, we demonstrated that the growth, infiltration and metastasis of malignant tumors require angiogenesis. As an important multi-functional angiogenesis factor, VEGF exerts its function by specifically acting on receptors on the vascular endothelial cell surface, which cannot only promote mitosis and proliferation of vascular endothelial cells but also enhance capillary permeability (23). Upregulated VEGF expression can be observed in all malignant tumors, and thus it plays a vital role in angiogenesis, infiltration and metastasis of tumors (24). To our surprise, we found that microRNA-26b significantly suppressed the NF-кB/MMP-9/VEGF pathway.
Matrix metalloproteinases (MMPs) are a class of zincdependent proteases which can degrade the extracellular matrix (ECM) as well as a majority of proteins on the basement membrane, and they play important roles in the invasion and metastasis of malignant tumors (11). MMP-9 belongs to the gelatinase family, which mainly degrades gelatin, along with type IV, V, VII and X basement membrane collagen (25). Its high expression can result in accelerated degradation of ECM and the basement membrane of blood vessels, stimulates tumor cells to move out of the carcinoma nest along with the injured basement membrane and infiltrate to surrounding tissues, which is benefit to tumor cells going in and out of blood vessels, thus promoting distal metastasis (26). High MMP-9 expression is related to the enhanced invasion and metastatic abilities of multiple tumors, including gastric, colon and liver cancer (22). Therefore, our results suggest that the inhibition of NF- $\kappa \mathrm{B}$ significantly enhanced the effects of microRNA-26b on suppression of the NF- $\mathrm{B} / \mathrm{MMP}-9 / \mathrm{VEGF}$ pathway, inhibition of cell proliferation, induction of apoptosis, and promotion of Bax and caspase-3/-9 protein expression in a liver cancer cell line. Li et al revealed that microRNA-26b suppresses nonsmall cell lung cancer metastasis through the NF- $\mathrm{KB} / \mathrm{MMP}-9 /$ VEGF pathway (27).

In conclusion, our findings indicate that microRNA-26b has anticancer effects, inhibits cell proliferation, induces apoptosis and promotes Bax and caspase-3/-9 protein expression in liver cancer cells mainly through the PI3K/Akt and NF- $\mathrm{B} /$ MMP-9/VEGF pathways (Fig. 12). Those findings suggest the microRNA-26b may be a novel biomarker for the early diagnosis or may aid in developing new clinical treatments for liver cancer.

\section{References}

1. Bush DA, Smith JC, Slater JD, Volk ML, Reeves ME, Cheng J, Grove R and de Vera ME: Randomized clinical trial comparing proton beam radiation therapy with transarterial chemoembolization for hepatocellular carcinoma: Results of an interim analysis. Int J Radiat Oncol Biol Phys 95: 477-482, 2016.

2. Sharma M, Tuaine J, McLaren B, Waters DL, Black K, Jones LM and McCormick SP: Chemotherapy agents alter plasma lipids in breast cancer patients and show differential effects on lipid metabolism genes in liver cells. PLoS One 11: e0148049, 2016.

3. Guo JR, Shen HC, Liu Y, Xu F, Zhang YW, Shao Y and Su YJ: Effect of acute normovolemic hemodilution combined with controlled low central venous pressure on blood coagulation function and blood loss in patients undergoing resection of liver cancer operation. Hepatogastroenterology 62: 992-996, 2015.

4. Han S, Tang Q, Lu X, Chen R, Li Y, Shu J, Zhang X and Cao J: Dysregulation of hepatic microRNA expression profiles with Clonorchis sinensis infection. BMC Infect Dis 16: 724, 2016.

5. He S, Zhang DC and Wei C: MicroRNAs as biomarkers for hepatocellular carcinoma diagnosis and prognosis. Clin Res Hepatol Gastroenterol 39: 426-434, 2015.

6. Tan Y, Lin B, Ye Y, Wen D, Chen L and Zhou X: Differential expression of serum microRNAs in cirrhosis that evolve into hepatocellular carcinoma related to hepatitis B virus. Oncol Rep 33: 2863-2870, 2015.

7. Feng X, Jiang J, Shi S, Xie H, Zhou L and Zheng S: Knockdown of miR-25 increases the sensitivity of liver cancer stem cells to TRAIL-induced apoptosis via PTEN/PI3K/Akt/Bad signaling pathway. Int J Oncol 49: 2600-2610, 2016.

8. Sui Y, Zheng X and Zhao D: Rab31 promoted hepatocellular carcinoma (HCC) progression via inhibition of cell apoptosis induced by $\mathrm{PI} 3 \mathrm{~K} / \mathrm{AKT} / \mathrm{Bcl}-2 / \mathrm{BAX}$ pathway. Tumour Biol 36: 8661-8670, 2015. 
9. Palian BM, Rohira AD, Johnson SA, He L, Zheng N, Dubeau L, Stiles BL and Johnson DL: Maf1 is a novel target of PTEN and PI3K signaling that negatively regulates oncogenesis and lipid metabolism. PLoS Genet 10: e1004789, 2014.

10. Zhang H, Zhao B, Huang C, Meng XM, Bian EB and Li J: Melittin restores PTEN expression by down-regulating HDAC2 in human hepatocelluar carcinoma HepG2 cells. PLoS One 9: e95520, 2014.

11. Shin SY, Kim CG, Jung YJ, Lim Y and Lee YH: The UPR inducer DPP23 inhibits the metastatic potential of MDA-MB-231 human breast cancer cells by targeting the Akt-IKK-NF- $\mathrm{BB}-\mathrm{MMP}-9$ axis. Sci Rep 6: 34134, 2016.

12. Manna K, Khan A, Kr Das D, Bandhu Kesh S, Das U, Ghosh S, Sharma Dey R, Das Saha K, Chakraborty A, Chattopadhyay S, et al: Protective effect of coconut water concentrate and its active component shikimic acid against hydroperoxide mediated oxidative stress through suppression of NF- $\mathrm{BB}$ and activation of Nrf2 pathway. J Ethnopharmacol 155: 132-146, 2014.

13. Moulton CA, Gu CS, Law CH, Tandan VR, Hart R, Quan D, Fairfull Smith RJ, Jalink DW, Husien M, Serrano PE, et al: Effect of PET before liver resection on surgical management for colorectal adenocarcinoma metastases: A randomized clinical trial. JAMA 311: 1863-1869, 2014.

14. Melucci E, Cosimelli M, Carpanese L, Pizzi G, Izzo F, Fiore F, Golfieri R, Giampalma E, Sperduti I, Ercolani C, et al; Italian Society of Locoregional Therapies in Oncology (S.I.T.I.L.O.): Decrease of survivin, $\mathrm{p} 53$ and $\mathrm{Bcl}-2$ expression in chemorefractory colorectal liver metastases may be predictive of radiosensivity radiosensivity after radioembolization with yttrium-90 resin microspheres. J Exp Clin Cancer Res 32: 13, 2013.

15. Gnoni A, Santini D, Scartozzi M, Russo A, Licchetta A, Palmieri V, Lupo L, Faloppi L, Palasciano G, Memeo V, et al: Hepatocellular carcinoma treatment over sorafenib: Epigenetics, microRNAs and microenvironment. Is there a light at the end of the tunnel? Expert Opin Ther Targets 19: 1623-1635, 2015.

16. Sun J, Zhou M, Yang H, Deng J, Wang L and Wang Q: Inferring potential microRNA-microRNA associations based on targeting propensity and connectivity in the context of protein interaction network. PLoS One 8: e69719, 2013.

17. He J, Zhao K, Zheng L, Xu Z, Gong W, Chen S, Shen X, Huang G, Gao M, Zeng Y, et al: Upregulation of microRNA-122 by farnesoid $\mathrm{X}$ receptor suppresses the growth of hepatocellular carcinoma cells. Mol Cancer 14: 163, 2015.

18. Park KU, Seo YS, Lee YH, Park J, Hwang I, Kang KJ, Nam J, Kim SW and Kim JY: Altered microRNA expression profile in hepatitis B virus-related hepatocellular carcinoma. Gene 573: 278-284, 2015
19. Zhang Y, Zheng L, Ding Y, Li Q, Wang R, Liu T, Sun Q, Yang H, Peng S, Wang W, et al: MiR-2Oa induces cell radioresistance by activating the PTEN/PI3K/Akt signaling pathway in hepatocellular carcinoma. Int J Radiat Oncol Biol Phys 92: 1132-1140, 2015.

20. Zhu A, Kang N, He L, Li X, Xu X and Zhang H: miR-221 and miR-26b regulate chemotactic migration of MSCs toward HGF through activation of Akt and FAK. J Cell Biochem 117: 1370-1383, 2016

21. Luo J, Zhou H, Wang F, Xia X, Sun Q, Wang R and Cheng B: The hepatitis $B$ virus $X$ protein downregulates NF- $\kappa B$ signaling pathways through decreasing the Notch signaling pathway in HBx-transformed L02 cells. Int J Oncol 42: 1636-1643, 2013.

22. Hsieh SC, Tsai JP, Yang SF, Tang MJ and Hsieh YH: Metformin inhibits the invasion of human hepatocellular carcinoma cells and enhances the chemosensitivity to sorafenib through a downregulation of the ERK/JNK-mediated NF- $\kappa$ B-dependent pathway that reduces uPA and MMP-9 expression. Amino Acids 46: 2809-2822, 2014.

23. Alidzanovic L, Starlinger P, Schauer D, Maier T, Feldman A, Buchberger E, Stift J, Koeck U, Pop L, Gruenberger B, et al: The VEGF rise in blood of bevacizumab patients is not based on tumor escape but a host-blockade of VEGF clearance. Oncotarget 7: 57197-57212, 2016.

24. Guo LY, Zhu P and Jin XP: Association between the expression of HIF- $1 \alpha$ and VEGF and prognostic implications in primary liver cancer. Genet Mol Res 15: 15, 2016.

25. Zheng CG, Chen R, Xie JB, Liu CB, Jin Z and Jin C: Immunohistochemical expression of Notch1, Jagged1, NF- $\kappa B$ and MMP-9 in colorectal cancer patients and the relationship to clinicopathological parameters. Cancer Biomark 15: 889-897, 2015.

26. Grünwald B, Vandooren J, Locatelli E, Fiten P, Opdenakker G, Proost P, Krüger A, Lellouche JP, Israel LL, Shenkman L, et al: Matrix metalloproteinase-9 (MMP-9) as an activator of nanosystems for targeted drug delivery in pancreatic cancer. J Control Release 239: 39-48, 2016.

27. Li D, Wei Y, Wang D, Gao H and Liu K: MicroRNA-26b suppresses the metastasis of non-small cell lung cancer by targeting MIEN1 via NF- $\mathrm{B} / \mathrm{MMP}-9 / \mathrm{VEGF}$ pathways. Biochem Biophys Res Commun 472: 465-470, 2016. 